\title{
Über die Autoren/Abstracts
}

\section{Stephan Elspaß}

Stephan Elspaß war 1995 bis 1997 DAAD-Lektor an der Aston University in Birmingham (GB) und ist seit 2004 Inhaber des Lehrstuhls für Deutsche Sprachwissenschaft an der Universität Augsburg. Seine Lehr- und Forschungsinteressen liegen in den Bereichen Sprachvariation und -wandel, Soziolinguistik, Neuere Sprachgeschichte, (Historische) Grammatik, Sprachpolitik und Phraseologie.

\section{Reinhard Fiehler}

Prof. Dr. phil.; 1969-1977 Studium der Fächer Germanistik und Soziologie an den Universitäten Hamburg, Freiburg und Osnabrück; 1977 Magister Artium und 1979 Promotion an der Universität Osnabrück mit der Arbeit Kommunikation und Kooperation; 1979-1990 Wissenschaftlicher Assistent an der Universität Bielefeld; 1987 Habilitation an der Fakultät für Linguistik und Literaturwissenschaft der Universität Bielefeld mit der Arbeit Kommunikation und Emotion; 1990-1994 Hochschuldozent an der Universität Bielefeld; seit 1994 Mitarbeiter des Instituts für deutsche Sprache; 1994 Ernennung zum außerplanmäßigen Professor an der Fakultät für Linguistik und Literaturwissenschaft der Universität Bielefeld.

\section{Andreas Jäger}

Dr. phil. (DaF); 2004 Prom. Universität Bielefeld. Von 1997-2000 Erstellung von Sprachlernsoftware und seit 1997 Autor und Redakteur für DaF-Lehrmaterialien für den Klett-Verlag. Von 2000-2005 DaFLehrer und stellvertretender Leiter am Sprachinstitut dialoge Lindau sowie Mitarbeit im EU-Projekt www.montanalingua.com. Seit 2006 DAAD-Lektor an der University of Sydney sowie Leiter des
DAAD-Information Centers Sydney. Forschung: Sprachenpolitik und -planung, Kultur- und Landeskunde sowie Methodik/Didaktik DaF.

\section{Sabine Jasny}

Dr. phil. (DaF); 2001 Prom. LMU München. Von 1996 bis 2005 Fachleitung und Lehrerin am Sprachinstitut dialoge Lindau. Daneben seit 1997 DaF-Autorin für den Klett-Verlag, von 2002-2004 Seminarleiterin in der Lehrerfortbildung am Goethe-Institut München sowie von 2003-2005 Antragstellung und Projektleiterin im EU-Projekt www.montanalingua.com. Im Jahr 2005 DaF-Dozentin an der Universität Bielefeld. Seit 2007 an der University of Sydney. Forschung: Deutsch als fremde Wissenschaftssprache, Sprachenpolitik und -planung sowie Methodik/Didaktik DaF.

\section{Tristan Lay}

Dr. phil., geb. 1978; Studium der Sprachlehrforschung und Sinologie in Bochum, M. A. 2002, 2004 Promotion am Seminar für Sprachlehrforschung an der RuhrUniversität Bochum. Seit 2006 Assistant Professor für Deutsch als Fremdsprache am Europäischen Institut für Fremdsprachliche Philologien der National Chengchi University in Taipeh. Forschungsschwerpunkte und Publikationen in den Bereichen Sprachlehrforschung und Deutsch als Fremdsprache.

\section{Péter Maitz}

Péter Maitz ist Oberassistent am Lehrstuhl für Germanistische Linguistik an der Universität Debrecen (Ungarn). 2000 bis 2002 war er Mitglied des DFG-Graduiertenkollegs »Dynamik von Substandardvarietäten« der Universitäten Hei- 
delberg und Mannheim und promovierte 2002 mit einer Arbeit über die Mehrsprachigkeit in der Habsburgermonarchie. Zur Zeit forscht und lehrt er als Humboldt-Stipendiat an der Universität Augsburg. Seine soziolinguistischen Forschungen betreffen vor allem Fragen der Sprachgeschichte, Sprachvariation, Mehrsprachigkeit, Namenkunde, Sprachtheorie sowie sprachlicher Ideologien.

\section{Claudia Riemer}

Prof. Dr. phil.; 1984-1991 Studium der Fächer Deutsch als Fremdsprache, Germanistik und Soziologie an der Universität Bielefeld; 1991-1996 Promotionsstudiengang Deutsch als Fremdsprache an der Universität Bielefeld; 2002 Habilitation an der Universität Hamburg im Fach Sprachlehrforschung; seit 2002 Professur für Deutsch als Fremd- und Zweitsprache an der Universität Bielefeld. Forschungsschwerpunkte: Sprachlehr- und -lernforschung/Zweitsprachenerwerbsforschung (mit Schwerpunkt auf individuellen Lernprozessen, affektiven und kognitiven Einflussfaktoren); Deutsch als Fremdsprache; For- schungsmethodologie in der empirischen Fremdsprachenforschung (mit Schwerpunkt auf qualitativer Forschung). Mitglied im Beirat des Fachverbands Deutsch als Fremdsprache, Mitglied im Vorstand der Deutschen Gesellschaft für Fremdsprachenforschung.

\section{Werner Roggausch}

Dr. phil., geb. 1948. Studium der Germanistik, Politik und Kunstgeschichte. DAAD Lektor in China 1983 bis 1987. Seit 1990 Mitglied der Redaktion von Info DaF und Mitarbeiter des DAAD.

\section{Jörg Schröder}

Dr. phil.; Studium der Germanistik, Sinologie und Deutsch als Fremdsprache an den Universitäten Trier, Bochum und Lanzhou. Tätigkeit in der Erwachsenenbildung für DaF, Lehrbeauftragter und Fachlehrer für DaF (Universität Trier), DAAD-Lektor an der Universität Wuhan (1994-2000), seit 2003 DAAD-Lektor an der Zhejiang Universität in Hangzhou. Forschungsschwerpunkte: Wirtschaftsdeutsch, Interkulturelle Kommunikation, mediengestützter Unterricht. 


\begin{tabular}{|c|c|c|c|}
\hline 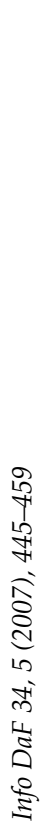 & 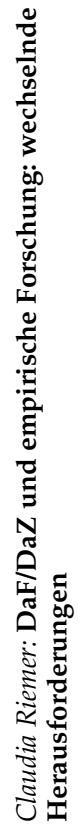 & 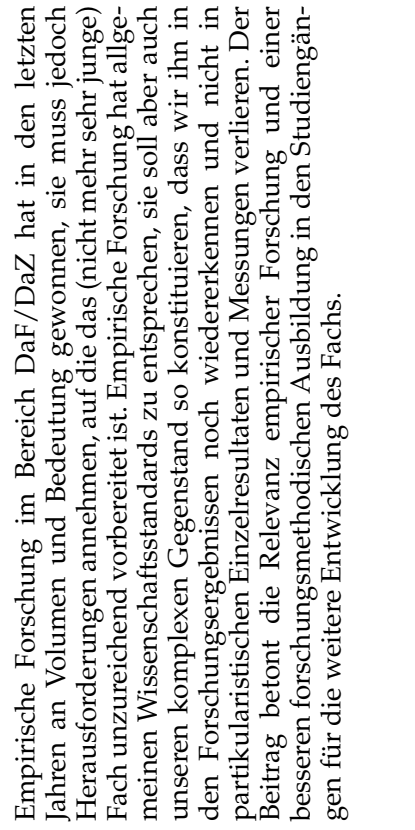 & 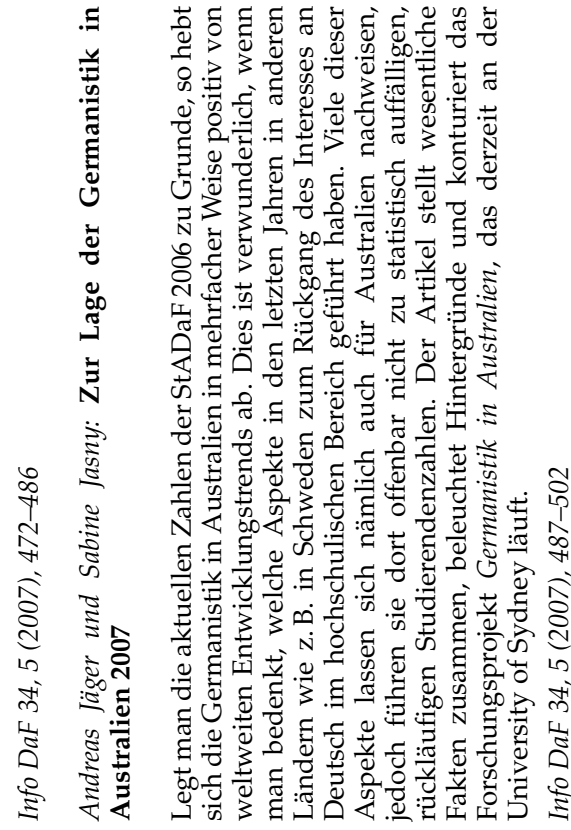 \\
\hline 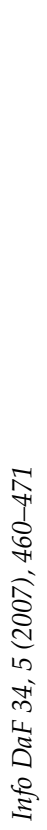 & 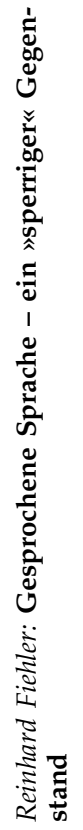 & 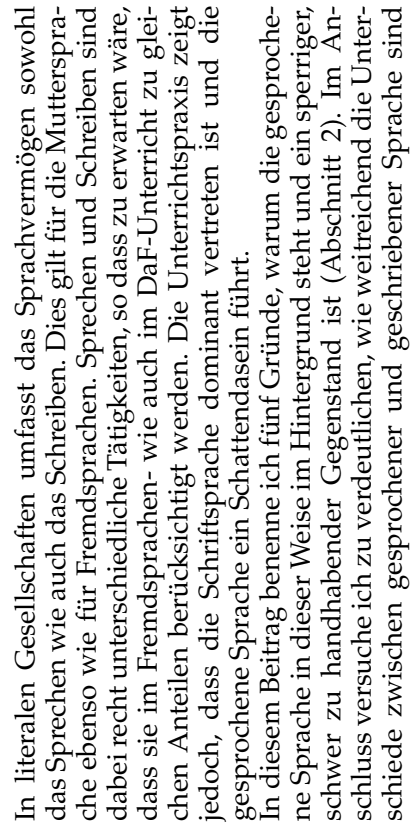 & 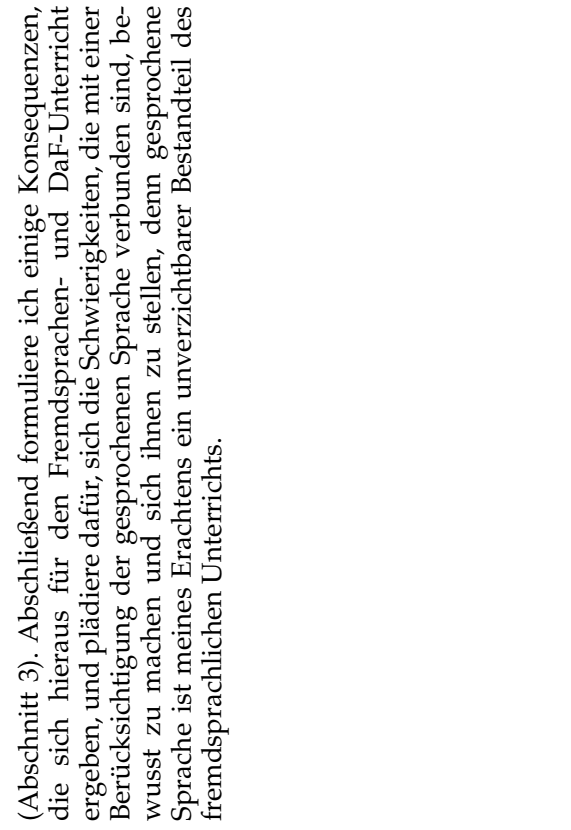 \\
\hline
\end{tabular}




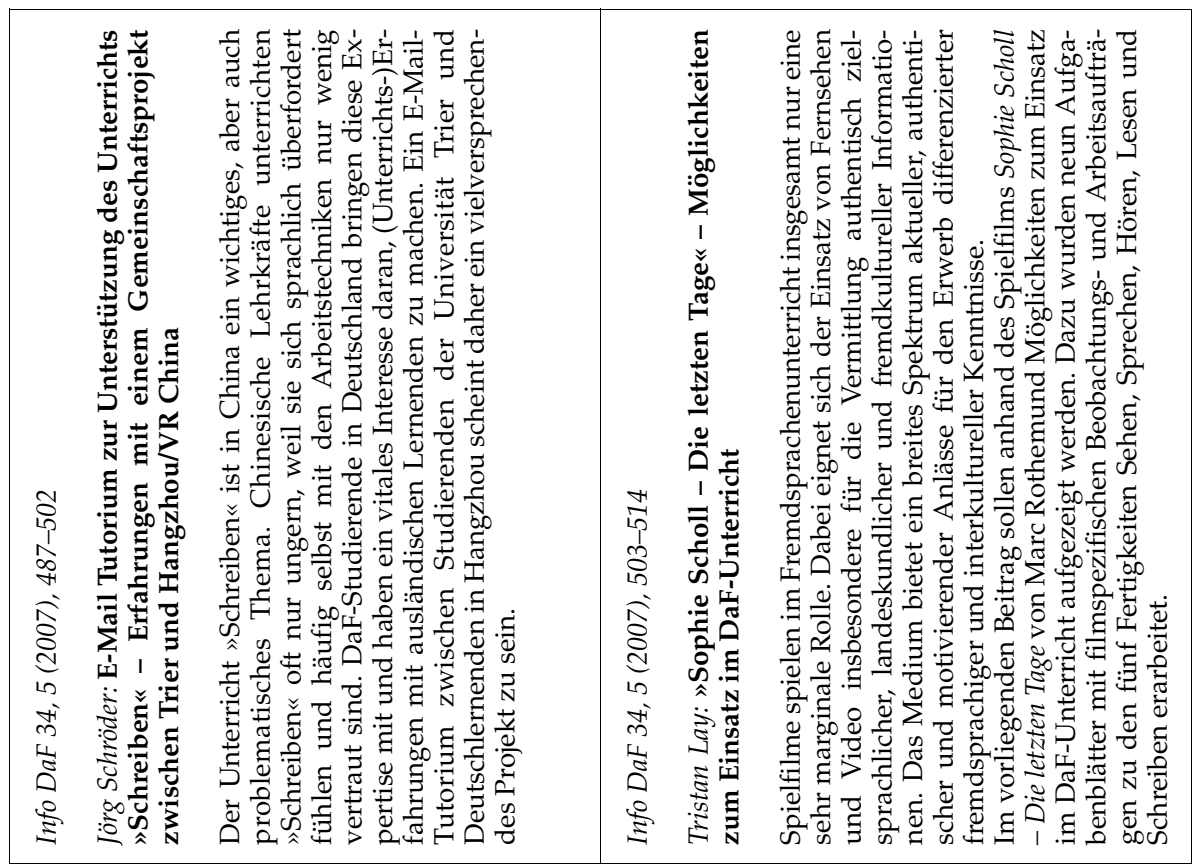

